\title{
Cognição Numérica de Crianças Pré-Escolares Brasileiras pela ZAREKI-K
}

\author{
Juliana Molina \\ Universidade Federal de São Paulo, São Paulo, SP, Brasil \\ Fabiana Silva Ribeiro \\ Escola de Psicologia da Universidade do Minho, Braga, Portugal \\ Flávia Heloísa Santos ${ }^{1 *}$ \\ Programa de Pós-Graduação em Psicologia do Desenvolvimento e Aprendizagem \\ da Universidade Estadual Paulista Júlio de Mesquita Filho, Bauru, SP, Brasil \\ Departamento de Psicologia Básica da Universidade do Minho, Braga, Portugal \\ Michael von Aster \\ Psychology Department at the University of Potsdam, Potsdam, Germany
}

\section{Resumo}

A Cognição Numérica é influenciada por fatores biológicos, cognitivos, educacionais e culturais. Constitui-se tanto de um sistema primário, chamado de Senso Numérico que seria inato e de abrangência universal, quanto de sistemas secundários, como o Cálculo que está implicado a realização de operações matemáticas por meio de símbolos ou palavras e o Processamento Numérico, que se divide em dois componentes, a Compreensão Numérica, que está relacionada ao entendimento de símbolos numéricos e a Produção Numérica: que inclui a leitura, escrita e contagem de números. Entretanto, os estudos que evidenciam o desenvolvimento destas funções em crianças de idade pré-escolar são escassos. Diante disso os objetivos do presente estudo foram investigar a cognição numérica em crianças brasileiras pré-escolares e demonstrar a validade de construto da ZAREKI-K (Bateria Neuropsicológica para Avaliação do Tratamento dos Números e do Cálculo para Crianças pré-escolares). Participaram da pesquisa 42 crianças, entre 5 e 6 anos de idade, de ambos os sexos, estudantes de escolas públicas de educação pré-escolar, que foram avaliadas pelos subtestes da ZAREKI-K e WISC-III. Os Resultados indicaram diferenças significativas associadas à idade em que crianças de 6 anos obtiveram melhores pontuações em subtestes relacionados a Produção numérica, Compreensão numérica e Cálculo, bem como correlações moderadas a fortes entre alguns subtestes de ambos os instrumentos, demonstrando a validade de constructo da bateria. Em conclusão, foram obtidos dados normativos preliminares para a ZAREKI-K, ademais as análises sugerem tratar-se de um instrumento promissor para a avaliação da cognição numérica em crianças pré-escolares.

Palavras-chave: Matemática, número, pré-escolares, memória operacional, Discalculia do Desenvolvimento.

Endereço para correspondência: Universidade Estadual Paulista Júlio de Mesquita Filho, Programa de PósGraduação em Psicologia do Desenvolvimento e Aprendizagem, Campus Bauru, Av. Engenheiro Luiz Edmundo Carrijo Coube, $n^{\circ}$ 14-01, Bauru, SP, Brasil 17033-360. Fone: + 5514 3103-6077. E-mail: flaviahs@, psi.uminho.pt

Agradecimentos: À Fundação de Amparo à Pesquisa do Estado de São Paulo (FAPESP) pelo Acordo de Cooperação Internacional, processo 04/11.067-1 coordenado pela Dra. Flávia Heloísa Santos, e respectivas bolsas de iniciação científica: Juliana Molina Processo no 05/00595, Ana Luiza Dias no 2005/00592-9, Bruna Pachoalini no 05/00594-1, Michele Frigério no 05/00593-5 e Paulo Adilson da Silva n ${ }^{\circ} 05 / 60375-1$. À Secretaria Municipal de Educação, diretoras e coordenadoras pedagógicas das escolas municipais da região centro-oeste paulista. Às crianças e aos seus pais que participaram do estudo. 


\title{
Numerical Cognition in Brazilian Preschool Children by the ZAREKI-K
}

\begin{abstract}
The Numerical Cognition is influenced by biological, cognitive, educational and cultural factors. It consists of a primary system, called Number Sense that would be innate and universal, also of secondary systems: the Calculation, implied to perform mathematical operations by means of symbols or words and Number Processing, which is divided into two components, Number Comprehension, related with the understanding of numerical symbols and Number Production, which includes reading, writing and counting numbers. However, studies that show the development of these functions in children of preschool age are scarce. Therefore, aims of this study were to investigate numerical cognition in preschool Brazilian children to demonstrate the construct validity of the ZAREKI-K (A Neuropsychological Battery for the Assessment of Treatment of Numbers and Calculation for preschool children). The participants were 42 children of both genders, who attended public elementary schools; the children were evaluated by this battery and WISC-III. The results indicated significant differences associated with age which children of 6 years had better scores on subtests related to Number Production, Calculation and Number Comprehension, as well moderate and high correlations between some subtests of both instruments, demonstrating the construct validity of the battery. In conclusion, preliminary normative data were obtained for ZAREKI-K. The analyses suggested that it is a promising tool for the assessment of numerical cognition in preschool children.
\end{abstract}

Keywords: Mathematics, number, preschoolers, working memory, Developmental Dyscalculia.

\section{Cognición Numérica de Niños Preescolares Brasileños por la ZAREKI-K}

\section{Resumen}

La cognición numérica es influenciada por factores biológicos, educativos, cognitivos y culturales. Posee un sistema primario innato y universal, que es el Sentido Numérico y sistemas secundarios: el Cálculo, para realizar operaciones matemáticas por medio de símbolos o palabras y el Procesamiento Numérico, que se divide en dos componentes, la Comprensión Numérica, que implica la comprensión de los símbolos numéricos, y la Producción Numérica que incluye la lectura, escritura y conteo de números. Sin embargo, hay pocos estudios que muestran el desarrollo de estos sistemas en los niños de edad preescolar. Los objetivos de este estudio fueran investigar la cognición numérica en niños brasileños en edad preescolar y demostrar la validez de constructo de la ZAREKI-K (Batería Neuropsicológica para la Evaluación del Tratamiento de los Números y el Cálculo para los Niños en edad Preescolar; Weinhold-Zulauf, Schweiter, \& von Aster, 2003). Los participantes fueron 42 niños de entre 5 y 6 años de edad, de ambos sexos, que estudiaban en escuelas públicas de educación preescolar, los niños fueron evaluados por la referida batería y la WISC-III. Los resultados indicaron que los niños de seis años presentaron mejores puntuaciones en las pruebas relacionadas con Producción Numérica, Cálculo y la Comprensión Numérica, así como las correlaciones altas y moderadas entre algunos subtest de ambos instrumentos, lo que demuestra la validez construirla batería. En conclusión, se obtuvieron datos preliminares normativos para el ZAREKI-K. Los análisis sugieren que este es un instrumento prometedor para la evaluación de la cognición numérica en niños preescolares.

Palabras clave: Matemática, número, preescolar, memória operativa, Discalculia do Desenvolvimento. 
Estudos demonstram que a cognição numérica - compreensão implícita de numerosidade, ordinalidade, contagem e aritmética simples -, está presente desde o início do desenvolvimento humano, como um sistema inato, biologicamente primário e presente em outras espécies animais, o que sugere sua abrangência universal (Cantlon, Platt, \& Brannon, 2008; Geary, 1995, 2000; Geary et al., 2009; Libertus, Feigenson, \& Halberda, 2011).

De acordo com Krajewski e Schneider (2009), os bebês recém-nascidos já seriam capazes de diferenciar visualmente pequenas quantidades, isso sugere que o processamento numérico primário é independente das habilidades linguísticas (Brannon, 2005; Gelman \& Butterworth, 2005; Wynn, 1992). Contudo, com a aquisição da linguagem as crianças passariam a expressar verbalmente a diferenciação de quantidades, utilizando palavras representativas, tais como "menos e mais" ou "muito e pouco", entre outras (Resnick, 1989).

Os experimentos evidenciam que esta capacidade para realizar julgamento numérico de desigualdades é determinada pela relação de ordem entre dois números quando eles são diferentes, por exemplo, "maior que" ou "menor que" (Moyer \& Landauer, 1967). Em outras palavras, as crianças pré-escolares conseguem discriminar quantidades, quando essas se diferem em uma relação equivalente ao dobro (por exemplo, $8 v s$. 4, 16 vs. 8 e 32 vs. 16), porém quando a relação é menor (por exemplo: 6 vs. 4, 12 vs. 8 e 24 vs. 16), falham na discriminação entre menor e maior, pois este ato depende fundamentalmente da relação entre quantidades (Sekuler \& Mierkiewicz, 1977). A capacidade para julgamento numérico pode predizer o desenvolvimento de habilidades quantitativas secundárias adquiridas via ensino formal (Mussolin, Nys, Content, \& Leybaert, 2014), as quais requerem a aprendizagem de relações precisas entre as quantidades (Xu, Spelke, \& Goddard, 2005).

A partir de cerca de dois anos de idade as crianças aprendem a pronunciar os números e a contar, bem como a interligar os números às palavras em sua exata sequência. No entanto, elas ainda não possuem a habilidade de empregar palavras com o objetivo de nomear os números de acordo com as quantidades que estes representam. Esse processo se desenvolve concomitantemente à consciência fonológica, já que essa contribui para a aquisição da sequência palavra-número, em relação às habilidades vísuoespaciais (Krajewski \& Schneider, 2009).

Segundo Dehaene (1997) o indivíduo nasce com uma espécie de acumulador, denominado Senso Numérico, que lhe permite estimar quantidades de vários tamanhos, embora apenas reconheça com maior precisão pequenos conjuntos de objetos. O senso numérico é uma habilidade para representar e manipular magnitudes numéricas não verbais, que explicaria a capacidade de julgamento numérico de desigualdades em bebês. Para Dehaene (2001), o senso numérico seria um sistema central que se ligaria às representações numéricas visuais e verbais para consolidar um alto nível de desenvolvimento aritmético, é suficientemente sofisticado para evitar enganos, caso ocorra remoção ou mudanças nas características de um objeto.

Desta maneira é notável a habilidade espontânea que as crianças apresentam para a capacidade de contagem, as quais possuem uma compreensão muito precoce da comutatividade da adição (por exemplo: a regra de que $a+b$ é sempre igual $a b+a)$. Estudos demonstraram que este princípio já pode ser observado em crianças de cinco anos de idade, desta forma a maioria das crianças na fase pré-escolar possui uma compreensão bem desenvolvida de aproximação e contagem (Kucian et al., 2011). Ao longo dos anos, as experiências da criança e a escolarização desenvolvem uma Linha Numérica Mental orientada espacialmente, que permite compreender rapidamente, aproximar e manipular quantidades numéricas maiores, convertendo as propriedades inatas da cognição numérica em habilidades secundárias.

McCloskey, Caramazza e Basili (1985) descreveram os sistemas secundários da cognição numérica - processamento numérico e cálculo, os quais se constituem de múltiplos componentes, modularmente organizados (Dehaene \& 
Cohen, 1995; McCloskey et al., 1985). O processamento numérico se refere à compreensão do símbolo numérico associado às suas quantidades, capacidade essa que estaria ligada ao senso numérico e aos primeiros estágios de desenvolvimento das habilidades matemáticas em pré-escolares e a produção numérica que inclui escrita, leitura e contagem numérica. Em contrapartida, a habilidade de cálculo se refere ao processamento dos símbolos ou palavras operacionais, à recuperação junto à memória de longo prazo de fatos aritméticos básicos (p. ex., tabuada) e à execução de procedimentos de cálculos aritméticos desenvolvidos mais tardiamente nas crianças (von Aster \& Shalev, 2007).

Consequentemente, a escola tem papel crucial no desenvolvimento dos sistemas secundários da cognição numérica por meio do ensino de técnicas aritméticas e na estruturação de ligações entre os mecanismos de cálculo e seu significado (Dehaene, 1997; Kucian et al., 2011). Por exemplo, Santos et al. (2012) observaram que crianças de 7 anos apresentavam dificuldades em tarefas de cálculo mental e problemas aritméticos, mas exibiam desempenho satisfatório em habilidades de compreensão de símbolos numéricos e estimativa perceptual, demonstrando que para habilidades relacionadas ao senso numérico a aprendizagem ocorre independente do ensino formal, entretanto para àquelas relacionadas ao Cálculo se verifica a necessidade de escolarização.

Dehaene e Cohen (1995) propuseram o modelo Código Triplo, no qual os números podem ser manipulados de três maneiras: uma representação analógica das quantidades, em que há ativação de uma linha mental para representação quantitativa dos números; um formato verbal, no qual os números são representados por uma ordem de palavras (p. ex., vinte três); e uma representação visual-arábica, na qual os números são representados por uma ordem de dígitos (p. ex., 23). A transcodificação, isto é, a passagem de um código numérico a outro, exemplo Arábico para verbal, pode ocorrer automaticamente. Além disso, cada procedimento de cálculo se apoia em um conjunto fixo de códigos de entrada e saída. O modelo postula que as tabelas de multiplicação são memorizadas por meio do código verbal, e que operações com múltiplos dígitos são realizadas mentalmente por meio do código visual arábico. Tarefas de comparação numérica ativariam o código de magnitude análoga, relacionando os números a uma linha mental de representação das magnitudes (Dehaene, 2001). Santos et al., (2012), utilizando uma bateria especializada para o estudo da cognição numérica em crianças escolares verificaram que a representação analógica, verbal e arábica visual dos números (código triplo) produz mudanças associadas à idade no desempenho aritmético.

Em 2007, von Aster e Shalev descreveram o Modelo de Desenvolvimento da Cognição Numérica em quatro passos; O Passo 1 - infância - consiste em um sistema central de representação numérica cardinal inata e funções adjacentes (subitizing - um processo automático para determinar a magnitude de um pequeno conjunto de itens - e aproximação), que provê o entendimento básico dos números. O Passo 2 - pré-escola - período em que as crianças aprendem a associar um número de objetos ou eventos a palavras ouvidas (representação verbal) e escritas, e no ensino fundamental as associam a dígitos sob a forma Arábica (Passo 3), com o desenvolvimento da simbolização numérica. No Passo 4, a linha numérica mental se desenvolve, a ordinalidade é adquirida, se tornando um segundo sistema central de representação numérica, cuja capacidade aumenta no decorrer dos anos, assim como a memória operacional. O Passo 1 é uma pré-condição para os Passos 2 e 3, e estes são pré-condições para o Passo 4. Este modelo é preditor de disfunções neurocognitivas em crianças com Discalculia do Desenvolvimento (Silva \& Santos, 2011).

Considerando este modelo, as crianças pré-escolares seriam naturalmente dotadas do passo 1 , ou seja, de uma representação interna para quantidades numéricas cujo desenvolvimento se inicia no primeiro ano de vida, em outras palavras, corresponde ao senso numérico que tardiamente subsidiará a aquisição de habilidades quantitativas secundárias. Durante suas experi- 
ências pré-escolares desenvolveriam a contagem de pelo menos 10 objetos e a capacidade de demonstração de conceitos simples como adição e subtração, tais conhecimentos que contribuiriam para o aprendizado gradual do passo 2, ou seja, do simbolismo da representação numérica e de suas relações preliminares (Sood \& Jitendra, 2011). Contudo, aspectos mais complexos, ligados aos passos 3 e, principalmente 4 , seriam adquiridos posteriormente, a partir da entrada da criança no ensino formal (Kercood, Zentall, Vinh, \& Tom-Wright, 2012).

Gilmore, McCarthy e Spelke (2010), a partir de dois experimentos envolvendo crianças pré-escolares e habilidades numéricas não simbólicas, tais como, a utilização de problemas de adição com números apresentados em matrizes de pontos e também com remoção de pontos de dentro de uma caixa; demonstraram que, embora a grade curricular não incluísse o conteúdo aritmético, as crianças obtiveram bons resultados em testes de adição e conhecimento numérico. Isto significa que a aprendizagem de todo processamento numérico simbólico no início da instrução formal da matemática depende das habilidades numéricas não simbólicas adquiridas antes mesmo do ingresso no ensino formal.

Weinhold-Zulauf, Schweiter e von Aster (2003), levando em consideração tanto o modelo do Código Triplo quanto o Modelo de Desenvolvimento da Cognição Numérica, desenvolveram a ZAREKI-K - Bateria Neuropsicológica para Avaliação do Tratamento dos Números e do Cálculo para Crianças pré-escolares (do alemão Neuropsychologische Testbatterie für Zahlenverarbeitung und Rechnen Bei Kindern, Kindergartenversion). A ZAREKI-K objetiva identificar e especificar o perfil das várias habilidades matemáticas básicas, necessárias para domínio do cálculo e da aritmética (Weinhold-Zulauf et al., 2003).

$\mathrm{O}$ estudo original desse instrumento, realizado em Zurique, na Suíça (Weinhold-Zulauf et al., 2003), demonstrou que as habilidades quantitativas primárias ou biológicas foram encontradas em crianças pré-escolares de 5 a 6 anos de idade e que estas, apresentaram mais dificuldades asso- ciadas as habilidades quantitativas secundárias, desenvolvidas pela entrada no ensino formal que envolvem a compreensão numérica e o cálculo. O estudo teve como foco a investigação sobre as diferenças relacionadas ao desempenho matemático de meninos e meninas em idade pré-escolar, além disso, estavam interessados ainda no efeito da idade sob a cognição numérica. Participaram do estudo 334 crianças, de 5 a 6 anos de idade, balanceadas por sexo $(51,2 \%$ meninas e meninos $48,8 \%$ ), as crianças foram avaliadas pela ZAREKI-K, os resultados demonstraram diferenças entre os grupos etários, sendo que as crianças de 6 anos, obtiveram desempenho superior às crianças de 5 anos. Diferenças quanto ao sexo, demonstraram que entre as crianças de 5 anos, foi observado melhor desempenho nas meninas para os seguintes subtestes (Contar; Problemas Matemáticos; Noção de Quantidade; e Transcodificação) em comparação com os meninos; entretanto, no grupo de 6 anos os meninos obtiveram melhor desempenho em quase todos os subtestes, com exceção de dois (Memória de Dígitos e Noção de Quantidade), segundo os pesquisadores esse fato pode ser devido a diferenças nas atitudes e estratégias de pensamento (verbal-analítico versus visual-espacial) entre meninos e meninas que se desenvolvem na infância. Contudo, são escassos os estudos que evidenciam o desenvolvimento da cognição numérica em crianças de idade pré-escolar de forma sistematizada, tal como pode ser proporcionado por esta bateria.

O presente estudo constitui a primeira pesquisa nacional utilizando a ZAREKI-K adaptada para a língua portuguesa e cultura brasileira, e teve por objetivo investigar a cognição numérica em crianças brasileiras pré-escolares. Foram objetivos específicos: (a) comparar o desempenho de crianças quanto ao sexo, idade e ambiente; (b) verificar possíveis relações entre subtestes da bateria e um instrumento pre-existente para estudar seu construto teórico.

Os autores apresentam as seguintes hipóteses: (a) crianças de 5 anos apresentarão desempenho inferior aos das crianças de 6 anos em habilidades quantitativas secundárias e (b) haverá diferenças associadas ao ambiente (rurais versus 
urbanas) e ao sexo (menino versus meninas) em subtestes específicos.

\section{Métodos}

\section{Aspectos Éticos}

O estudo foi aprovado pelo Comitê de Ética da Universidade Estadual Paulista "Júlio de Mesquita Filho", processo n. 0311/05.

\section{Participantes}

Foram avaliadas 42 crianças balanceadas quanto a idade e ao sexo, com idade de 5 a 6 anos, sendo, 19 de regiões rurais e 23 de regiões urbanas do centro-oeste paulistano. Foram utilizados como critérios de inclusão o nível intelectual médio aferido pelo teste Matrizes progressivas de Raven (percentil $>26$ e $<75$; Angelini, Alves, Custódio, Duarte, \& Duarte, 1999), crianças matriculadas em escolas públicas para educação pré-escolar. Este estudo foi realizado antes da mudança curricular, por este motivo as crianças de 6 anos não frequentavam o ensino formal para alfabetização. Crianças rurais e urbanas frequentavam as mesmas escolas, portanto, estavam submetidas às mesmas metodologias de ensino e currículo pedagógico adotados na rede pública municipal. Os critérios de exclusão foram evidências de patologias neurológicas, psiquiátricas, subnutrição, transtornos de aprendizagem ou de comportamento e uso de substâncias psicoativas, rastreados por meio de entrevista de anamnese junto ao responsável pela criança. Conforme a Escala para Avaliação do Status Socioeconômico da Associação Brasileira dos Institutos de Pesquisa de Mercado (ABIPEME; Almeida \& Wickerhauser, 1991), houve equivalência quanto nível socioeconômico das famílias.

\section{Procedimentos}

As crianças foram selecionadas por meio de reunião escolar com os pais/responsáveis para explicação dos objetivos e procedimentos do estudo. Durante a reunião, os pais assinaram o Termo de consentimento livre e esclarecido e responderam à anamnese, questionário de aspectos socioeconômicos e qualidade de vida. Após esta etapa, as crianças foram avaliadas individualmente, na própria escola. As avaliações neuropsicológicas duraram em média 40 minutos, os instrumentos foram apresentados em ordem semi-aleatória intercalando tarefas verbais e não verbais sempre que possível para evitar efeito de fadiga.

\section{Materiais}

Anamnese. Foi utilizada para o rastreio dos critérios de exclusão; desenvolvida por Santos (2002), consiste de um roteiro de perguntas para avaliar aspectos gerais e específicos do desenvolvimento da criança nas dimensões: social, educacional, psicológica e de saúde.

ABIPEME: Escala para Avaliação do Status Socioeconômico da Associação Brasileira dos Institutos de Pesquisa de Mercado (Almeida \& Wickerhauser, 1991). Esta escala avalia o status socioeconômico da família da criança e o subdivide em categorias correspondentes às classes sociais, A, B, C, D, E, sendo A o nível socioeconômico mais elevado [35-46 pontos] e E o nível mais baixo [0-4 pontos].

EGQV: Escala Global de Qualidade de Vida (Casas-Fernández, 1997). Constituído de 5 perguntas relacionadas ao comportamento, aprendizagem, autonomia, relações sociais e qualidade de vida. Cada pergunta oferece de 4 a 5 alternativas de respostas indicando do menor para o maior grau de autonomia.

Matrizes Progressivas Coloridas de Raven - Escala Especial (Angelini et al., 1999). É um teste padronizado que avalia a capacidade educativa que está relacionada a capacidade de extrair significado e desenvolver novas compreensões, constituído por três séries de doze itens que aumentam progressivamente em grau de dificuldade: A. identidade e mudança em padrões contínuos, $\mathrm{Ab}$. figuras distintas com todos espacialmente relacionados e $\mathrm{B}$. mudanças análogas em figuras relacionadas espacialmente e logicamente. No presente estudo, o teste foi utilizado na forma de caderno com gravuras coloridas. Cada item do teste apresenta na mesma folha 
uma figura incompleta e seis opções de resposta. A criança deve escolher apenas uma das alternativas para completar a figura.

WISC-III: Escala de Inteligência de Wechsler para Crianças - 3. edição (Wechsler, 2002; Adaptação e padronização para uma amostra brasileira por Figueiredo, 2002). Foram utilizados seis subtestes:

1. Vocabulário: a criança deve conceituar oralmente cada palavra lida em voz alta pelo examinador. Após 6 erros o subteste é interrompido;

2. Aritmética: a criança deve resolver uma série de problemas aritméticos. Para cada item, a criança resolve o problema mentalmente, sem usar lápis ou papel, e responde oralmente de acordo com o limite de tempo. Após 3 erros o subteste é interrompido;

3. Semelhanças: para cada item são apresentadas duas palavras oralmente que representam objetos ou conceitos comuns. A criança deve dizer o que estes dois objetos ou conceitos têm em comum. Após 8 erros o subteste é interrompido;

4. Dígitos: o examinador lê em voz alta uma sequência de números. Para cada item de dígitos na Ordem Direta, a criança deve repetir os números na mesma ordem em que foram falados. Para cada item na Ordem Inversa, a criança repete os números na ordem inversa. Cada item apresenta duas tentativas e cada tentativa possui o mesmo número de dígitos, mas os números são diferentes. $\mathrm{O}$ subteste é interrompido depois de haver erro nas duas tentativas de um mesmo item.

5. Cubos: Pede-se ao sujeito que reproduza, com cubos de faces coloridas, desenhos que lhe são mostrados. O subteste é interrompido após dois erros consecutivos;

6. Arranjo de Figuras: A criança deve analisar alguns conjuntos de figuras que lhe são apresentados e, organizá-las de forma que uma história lógica seja formada. O subteste é interrompido após seis erros consecutivos.

ZAREKI-K: A Bateria Neuropsicológica para Avaliação do Tratamento dos Números e do Cálculo para Pré-Escolares. É composta de
9 subtestes que avaliam a Cognição Numérica tanto em habilidades primárias quanto secundárias, por meio de tarefas apropriadas para crianças desta faixa etária. A consistência interna para a amostra mensurada pelo alpha de Cronbach foi de 0,93 (Weinhold-Zulauf et al., 2003). A ZAREKI-K original é protegida por direitos autorais (C) 2003 pela Pearson Assessment in Germany. A tradução e adaptação ao Português Brasileiro foi desenvolvida com o conhecimento da editora em território nacional e com a colaboração ativa do autor Michael von Aster, durante o Acordo de Cooperação Internacional financiado pela FAPESP.

São subtestes do ZAREKI-K:

A. Contar: Esta tarefa objetiva que as crianças desempenhem tarefas de contagem que envolvem: contagem direta, na ordem inversa, contagem em pares, antecessor e sucessor e contagem concreta de figuras. Esta tarefa avalia a capacidade do processamento numérico especificadamente a Produção $\mathrm{Nu}$ mérica.

B. Problemas Matemáticos: A criança é solicitada a realizar problemas numéricos mentalmente com dificuldade crescente, que incluem contas de adição e subtrações. $\mathrm{O}$ componente avaliado é a capacidade para o Cálculo.

C. Memorização de dígitos: Nesta tarefa é requerida que a criança apenas repita verbalmente uma série de números de dificuldade crescente, esta tarefa está relacionada ao Span em ordem direta, visto que a capacidade da memória é essencial para o Processamento numérico e o Cálculo.

D. Adição/Subtração: A criança realizará duas tarefas de cálculo concreto e outra oral, ambas contendo atividades de adição e subtração. Procura estimar a habilidade para o componente relacionado ao Cálculo.

E. Ordenar Números em uma Escala: A criança realizará duas tarefas que envolvem identificação numérica concreta advinda de informação verbal e por meio de informação escrita em número arábico, dentro de escalas verticais linhas horizontais em vários níveis. Esta tarefa é essencial para avaliação 
de componentes secundários como a linha numérica mental.

F. Noção de Quantidade - Parte perceptiva: A criança realizará uma série de tarefas de estimativa de quantidade com objetos concretos nos seguintes parâmetros: compreensão imediata, avaliação de estimativa, comparação e permanência de quantidades, esta tarefa avalia a capacidade do Senso Numérico.

G. Transcodificação: A criança realizará subtestes que envolvem a transcodificação de informações verbais para escrita, assim como de visual para a oral por meio de tarefas de leitura de números arábicos, escrita de números ditados verbalmente e ordenação de números e quantidades. Esses avaliam a capacidade de compreensão numérica, especificadamente o componente para a Produção Numérica.

H. Noção de quantidade - Parte cognitiva: A criança concretizará uma tarefa para avaliação contextual dos numerais orais, por meio das palavras "pouco", "médio" ou "bastante". Este subteste examina a habilidade de atribuir uma quantidade que depende do contexto e não do valor numérico, portanto avalia a compreensão numérica.

I. Comparação de quantidade: Pede-se à criança que realize uma série de tarefas que envolvem a capacidade simbólica numérica para feitos de comparação oral, escrita, espacial e de acordo com a magnitude dos números apresentados. Esta tarefa também avalia a capacidade da compreensão numérica.

\section{Análise Estatística}

Os dados obtidos foram analisados inicialmente visando à inspeção qualitativa dos dados, realizou-se primeiramente a análise descritiva, com médias e desvios-padrão. Para a análise com propostas inferenciais sobre os escores obtidos nos diversos testes neuropsicológicos foi realizada uma comparação (teste $t$ e magnitude de efeito) tendo como variáveis independentes o ambiente (rural versus urbano), a idade (5 versus 6 anos) e o sexo (masculino versus feminino). Os contrastes foram realizados pelo teste post- -hoc de Tukey e o nível de significância adotado foi o de $5 \%$.

Finalmente, foi realizado o coeficiente de correlação (Pearson) entre os escores obtidos nos subtestes da ZAREKI-K e os subtestes cubos, vocabulário, dígitos ordem direta e inversa, semelhanças, arranjo de figuras e aritmética do WISC III. Foram discutidas somente correlações moderadas $(r>0,4)$ e fortes $(r>0,7)$. Os dados obtidos foram analisados utilizando o software do STATISTICA - versão 7, Statsoft Incorporation 1984-2004 (StatSoft, 2004).

\section{Resultados}

As análises realizadas por meio do teste $t$ não evidenciaram diferenças significativas para as variáveis: ambiente e sexo, o que possibilitou desta forma, que os participantes fossem agrupados para comparações quanto à idade, sendo que a média dos grupos quanto ao status socioeconômico foi de $38( \pm 13)$, correspondente à classe $\mathrm{C}$. $\mathrm{O}$ percentil médio dos grupos nas Matrizes Progressivas de Raven foi de 54,75 ( \pm 14,18), classificado como intelectualmente médio (vide Tabela 1).

A Tabela 2 apresenta os resultados do teste $t$ para faixa etária (5 anos versus 6 anos) para os subtestes do WISC III e respectivos itens da ZAREKI-K. Diferenças significativas foram encontradas para os seguintes subtestes: A. Contar $(p=0,0003), \mathrm{B}$. Problemas matemáti$\cos (p=0,004)$; G- Transcodificação $(p=0,002)$; I-comparar quantidades $(p=0,002)$ e Total ZAREKI-K $(p=0,001)$, em que os escores das crianças de 6 anos de idade foram significativamente maiores do que das crianças com 5 anos de idade.

Foi realizada ainda a análise da magnitude de efeito utilizando a faixa etária como variável independente: (5 versus 6 anos de idade), as variáveis dependentes foram os subtestes da ZAREKI-K. De acordo com o Cohen' $d$ foram encontrados efeitos de grande magnitude nos subtestes: A $(d=$ $0,9), \mathrm{B}(d=0,9), \mathrm{E}(d=0,8), \mathrm{G}(d=0,9), \mathrm{I}(d=$ $0,9)$ e para ZAREKI-K escore total $(d=0,9)$ em que o desempenho das crianças de 6 anos de idade foram superiores aos das crianças de 5 anos. 
Tabela 1

Características Amostrais, quanto a Sexo, Ambiente, Inteligência, Qualidade de Vida e Aspectos Socioeconômicos

\begin{tabular}{lcccc}
\hline & $\begin{array}{c}5 \text { anos } \\
(n=21)\end{array}$ & $\begin{array}{c}6 \text { anos } \\
(n=21)\end{array}$ & $t$ & $p$ \\
\hline Sexo, $N(\%$ de meninos) & $11(52 \%)$ & $10(48 \%)$ & $-0,30$ & 0,76 \\
Ambiente, $N(\%$ urbanos) & $23(54 \%)$ & $19(45 \%)$ & $-0,30$ & 0,76 \\
Matrizes Progressivas de Raven, $M(D P)$ & $51,9(13,2)$ & $55,2(14,8)$ & $-0,77$ & 0,44 \\
Escala global de qualidade de vida, $M(D P)$ & $19,4(2,5)$ & $19,3(1,9)$ & 0,15 & 0,87 \\
ABIPEME, $M(D P)$ & $38,1(14,0)$ & $38,9(14,1)$ & $-0,17$ & 0,86 \\
\hline
\end{tabular}

Notas. Legenda: $N=$ número de participantes; $M=$ média; $D P=$ desvio-padrão; $t=$ teste $t ; p=$ significância; $\mathrm{ABIPEME}=$ Escala para Avaliação do Status Socioeconômico da Associação Brasileira dos Institutos de Pesquisa de Mercado.

Tabela 2

Escores [média (DP)] dos Resultados por Idades Obtidos em cada Subtotal da ZAREKI-K E Subtestes do WISC III

\begin{tabular}{|c|c|c|c|c|c|}
\hline Subtestes da ZAREKI-K & $\begin{array}{l}5 \text { anos } \\
(N=21)\end{array}$ & $\begin{array}{c}6 \text { anos } \\
(N=21)\end{array}$ & $t$ & $p$ & $d$ \\
\hline A: Contar & $15,5(1,5)$ & $23,7(1,5)$ & $-3,90$ & $0,0003 *$ & $-0,9 \#$ \\
\hline B: Problemas matemáticos & $1,1(0,4)$ & $3,0(0,4)$ & $-2,97$ & $0,004^{*}$ & $-0,9 \#$ \\
\hline $\mathrm{C}$ : Repetir números & $2,7(1,9)$ & $3,4(1,4)$ & $-1,34$ & 0,18 & $-0,2$ \\
\hline D: Adição/subtração & $9,0(1,3)$ & $12,3(1,3)$ & $-1,70$ & 0,09 & $-0,7$ \\
\hline E: Ordenar números em uma escala & $3,5(0,3)$ & $4,3(0,3)$ & $-1,65$ & 0,10 & $-0,8 \#$ \\
\hline F: Noção de quantidade - perceptiva & $7,8(0,6)$ & $8,3(0,6)$ & $-0,60$ & 0,55 & $-0,3$ \\
\hline G: Transcodificação & $8,5(0,9)$ & $12,7(0,9)$ & $-3,29$ & $0,002 *$ & $-0,9 \#$ \\
\hline H: Noção de quantidade - cognitiva & $3,0(0,2)$ & $3,3(0,2)$ & $-0,94$ & 0,35 & $-0,5$ \\
\hline I: Comparar quantidades & $21,9(0,9)$ & $26,0(0,9)$ & $-3,28$ & $0,002 *$ & $-0,9 \#$ \\
\hline Total ZAREKI-K & $73,4(4,8)$ & $97,0(4,8)$ & $-3,49$ & $0,001 *$ & $-0,9 \#$ \\
\hline \multicolumn{6}{|l|}{ Subtestes do WISC III } \\
\hline Cubos & $6,6(4,8)$ & $8,3(5,0)$ & 1,8 & 0,07 & $-0,1$ \\
\hline Vocabulário & $9,2(3,6)$ & $12,4(3,2)$ & 2,6 & 0,06 & $-0,4$ \\
\hline Dígitos Ordem Direta & $3,9(1,7)$ & $4,6(1,0)$ & $-0,005$ & 0,99 & $-0,2$ \\
\hline Dígitos Ordem Inversa & $1,0(1,1)$ & $2,0(1,4)$ & 2,26 & 0,08 & $-0,3$ \\
\hline Semelhanças & $3,1(2,1)$ & $3,9(1,7)$ & 1,4 & 0,15 & $-0,2$ \\
\hline Arranjo de Figuras & $5,1(4,7)$ & $7,6(6,3)$ & 2,17 & 0,91 & $-0,2$ \\
\hline Aritmética & $6,0(3,5)$ & $8,8(2,6)$ & 0,5 & 0,61 & $-0,4$ \\
\hline
\end{tabular}

Notas. Legenda: ZAREKI-K: A Bateria Neuropsicológica para Avaliação do Tratamento dos Números e do Cálculo para Crianças pré-escolares; WISC III: Escala de Inteligência de Wechsler para Crianças. Cohen's $d=0,2 ; 0,5$ e 0,8 respectivamente magnitude pequena, média ou grande.

$* p<0,05 ; \# d>0,8$. 
Foi realizada a correlação de Pearson entre os subtestes da ZAREKI-K e os subtestes do WISC III- Cubos, Vocabulário, Dígitos (ordem direta e inversa), Semelhanças, Arranjos de Figuras e Aritmética e observaram-se diversas correlações significativas (ver Tabela 3), dentre as quais destacamos as correlações moderadas entre os subtestes: (a) Contar com Dígitos Ordem Inversa, Arranjo de Figuras e Aritmética; (b) Problemas Aritméticos com Dígitos Ordem Inversa e Aritmética; (c) Repetir Números com
Dígitos Ordem Direta, Dígitos Ordem Inversa, Aritmética, (d) Adição/Subtração com Dígitos Ordem Inversa, Arranjo de Figuras e Aritmética; (e) Transcodificação com Dígitos Ordem Direta, Dígitos Ordem Inversa, Arranjo de Figuras e Aritmética; (f) Noção de quantidade-cognitiva com Dígitos Ordem Direta, Dígitos Ordem Inversa, Arranjo de Figuras e Aritmética, Subteste I com Dígitos Ordem Inversa, Arranjo de Figuras e Aritmética; (g) ZAREKI-K escore total com Vocabulário, Dígitos Ordem Direta, Dígitos Ordem Inversa, Arranjo de Figuras e Aritmética.

Tabela 3

Correlações entre ZAREKI-K e Subtestes do WISC III

\begin{tabular}{lcccccccccc}
\hline Testes & $\mathrm{A}$ & $\mathrm{B}$ & $\mathrm{C}$ & $\mathrm{D}$ & $\mathrm{E}$ & $\mathrm{F}$ & $\mathrm{G}$ & $\mathrm{H}$ & $\mathrm{I}$ & $\begin{array}{c}\text { Total } \\
\text { ZAREKI-K }\end{array}$ \\
\hline Cubos & $0,31^{*}$ & & & & & & & $0,38^{*}$ & & \\
$\begin{array}{l}\text { Vocabulário } \\
\text { Dígitos }\end{array}$ & $0,37^{*}$ & & $0,36^{*}$ & $0,38^{*}$ & & & $0,38^{*}$ & $0,32^{*}$ & $0,41^{*}$ & $0,47 \dagger$ \\
$\begin{array}{l}\text { Ordem Direta } \\
\text { Dígitos }\end{array}$ & & $0,39^{*}$ & $0,62 \dagger$ & $0,32^{*}$ & & & $0,51 \dagger$ & & & $0,42 \dagger$ \\
$\begin{array}{l}\text { Ordem Inversa } \\
\text { Semelhanças }\end{array}$ & $0,56 \dagger$ & $0,41 \dagger$ & $0,44 \dagger$ & $0,63 \dagger$ & & $0,32^{*}$ & $0,48 \dagger$ & $0,36^{*}$ & $0,57 \dagger$ & $0,66 \dagger$ \\
$\begin{array}{l}\text { Arranjo } \\
\text { de Figuras }\end{array}$ & $0,50 \dagger$ & $0,30^{*}$ & $0,37^{*}$ & $0,56 \dagger$ & $0,35^{*}$ & $0,32^{*}$ & $0,45 \dagger$ & $0,43 \dagger$ & $0,56 \dagger$ & $0,62 \dagger$ \\
Aritmética & $0,77 \dagger$ & $0,65 \dagger$ & $0,46 \dagger$ & $0,69 \dagger$ & & $0,34^{*}$ & $0,72 \dagger$ & $0,55 \dagger$ & $0,65 \dagger$ & $0,83 \dagger$ \\
\hline
\end{tabular}

Notas. Legenda: WISC III = da Escala de Inteligência de Wechsler para Crianças; Bateria ZAREKI-K: Bateria Neuropsicológica para Avaliação do Tratamento dos Números e do Cálculo para Crianças Pré-Escolares; $\mathrm{A}=$ Contar; $\mathrm{B}=$ Problemas Matemáticos; $\mathrm{C}=$ Repetir Números; $\mathrm{D}=$ Adição/Subtração; $\mathrm{E}=$ Ordenar números em uma escala; $\mathrm{F}=$ Noção de quantidade - parte perceptiva; $\mathrm{G}=$ Transcodificação; $\mathrm{H}$ = Noção de quantidade - parte cognitiva; $\mathrm{I}=$ Comparar quantidades.

${ }^{*} p<0,01 ; \dagger p<0,001, n=42$ (crianças de 5 anos e 6 anos de idade).

\section{Discussão}

O presente estudo investigou os sistemas de cognição numérica em crianças pré-escolares entre 5 e 6 anos de idade, além de demonstrar a validade de construto da ZAREKI-K (Bateria de testes neuropsicológicos para processamento de números e cálculo em crianças pré-escolares), considerando variáveis como idade, sexo e ambiente.

Com relação ao efeito de idade, tanto as crianças de 5 quanto as de 6 anos apresentaram desempenho similar nos subtestes relacionados as habilidades quantitativas primárias. Obser- vou-se que a criança pode realizar o subteste F- Noção de Quantidade, sem a necessidade de escolarização ou conhecimentos sobre símbolos matemáticos por meio do sistema Senso Numérico com o qual atribui valores e/ou efetua diferenciações de quantidades (Dehaene, 1997; Geary, 1995). Por outro lado, em habilidades quantitativas secundárias as crianças de 6 anos de idade exibiram melhor desempenho que as crianças de 5 anos: A - Contar e $\mathrm{G}$ - Transcodificação (Produção numérica), B - Problemas matemáticos (Cálculo), e I - Comparação de quantidades (Compreensão numérica) e na pontuação total. Portanto, há evidências de ganhos associados à 
idade em domínios do Processamento Numérico dependentes dos sistemas simbólicos de representação numérica (verbal e arábica) e Cálculo como um alicerce do sistema de magnitude ordinal, ainda incipiente, durante a fase pré-escolar (Dellatolas, von Aster, Braga, Meier, \& Deloche, 2000; Koumoula et al., 2004; von Aster \& Shalev, 2007). Isso significa que as habilidades matemáticas são dependentes tanto de escolaridade (von Aster \& Shalev, 2007), quanto de maturação cerebral (von Aster, 2000).

Estes resultados corroboram com Gilmore et al., (2010), que demonstraram que há uma relação específica entre o desempenho das crianças no desenvolvimento de habilidades aritméticas não simbólicas e nas habilidades numéricas simbólicas no início da escolaridade e antes mesmo de qualquer instrução formal em aritmética. O presente estudo sugere que elementos rudimentares dos diferentes sistemas de cognição numérica estão presentes precocemente, embora, conforme o modelo de von Aster e Shalev (2007) a aquisição de competência nos sistemas da Cognição Numérica ocorra paulatinamente ao desenvolvimento neurocognitivo e acadêmico (Dehaene, 1997).

Os nossos resultados gerais são similares ao estudo de Weinhold-Zulauf et al., (2003), porém, com diferenças sutis em subtestes específicos, por exemplo, crianças de 5 anos brasileiras obtiveram melhor desempenho que as crianças suíças na subteste I- Contar. Nos subtestes CMemória de dígitos, D- Adição/Subtração e EOrdenar números em uma escala, o desempenho das crianças foi semelhante, enquanto que nos subteste B- Problemas matemáticos e F- Noção de quantidades, as crianças suíças tiveram desempenho superior às crianças brasileiras. Estas discrepâncias podem refletir diferenças quanto aos fatores socioculturais e educacionais (Santos et al., 2012), bem como linguísticos (von Aster \& Shalev, 2007), os quais podem influenciar tanto o processamento numérico quanto o cálculo.

Em contraposição às nossas hipóteses e ao estudo de Weinhold-Zulauf et al., (2003), no que concerne ao efeito de gênero, não foram observadas diferenças entre meninos e meninas em nenhuma das tarefas, possivelmente devido a amostra, apesar de pequena, ser homogênea. Além disso, as crianças não apresentaram diferenças associadas ao ambiente, o que pode ser explicado pelo fato de que tanto as crianças urbanas quanto as rurais frequentavam as mesmas escolas localizadas na região urbana das cidades, beneficiando-se, portanto, do mesmo método pedágogico, resultado similar foi demonstrado previamente em escolares rurais e urbanos (Santos et al., 2012).

Foram encontradas correlações positivas moderadas e fortes entre os subtestes da ZAREKI-K e o subteste aritmética do WISC-III, as quais demonstram a validade do constructo da bateria, com a vantagem do primeiro em relação ao segundo no que se refere à discriminação de habilidades específicas inerentes à Cognição Numérica. Também foram encontradas correlações, entre os seguintes subtestes da ZAREKI-K: BProblemas Matemáticos e os seguintes subtestes do WISC-III; Vocabulário, Dígitos, Arranjo de figuras e Aritmética, demonstrando que o cálculo mental dependeria ainda de outras habilidades cognitivas como compreensão verbal, memória operacional e organização perceptual para sua execução (von Aster \& Dellatolas, 2006). O subteste I- Comparação de quantidades apresentou correlações moderadas com o teste Vocabulário, Dígitos ordem inversa, Arranjo de figuras e correlação forte com Aritmética, demonstrando que o desenvolvimento da compreensão numérica é subsidiado por outras habilidades cognitivas verbais relacionadas à análise, processamento e síntese da informação.

O estudo apresenta limitações no que concerne ao tamanho amostral e regionalismo, aspectos que restringem a generalização dos resultados obtidos, contudo, as diferenças significativas foram corroboradas pela magnitude do efeito, além disso, variáveis que poderiam influenciar nos resultados, tais como nível socioeconômico e intelectual, e aquelas descritas no critério de exclusão - foram devidamente controladas; tornando os resultados do estudo confiáveis.

Em conclusão, o desempenho da ZAREKI-K foi associado ao desenvolvimento (idade), corroborando com estudos prévios. Entretanto, pelo menos na presente amostra, o desempenho 
não foi influenciado por fatores ambientais (rural $\mathrm{x}$ urbano) e nem evidenciou diferenças associadas ao sexo. As análises de correlação com subtestes do WISC-III indicaram que habilidades cognitivas específicas como percepção, linguagem e memória operacional contribuem para o desenvolvimento da cognição numérica, ademais a principal correlação obtida entre o subteste de Aritmética do WISC-III e o escore total da bateria $(r=0,83)$, este dado demonstra a validade do constructo da bateria. Desta forma, o presente estudo obteve dados normativos preliminares para a ZAREKI-K, e de acordo com as análises apresentadas trata-se de um instrumento promissor para a avaliação de habilidades matemáticas em crianças pré-escolares. Cabe mencionar que novos estudos são necessários, controlando as limitações referidas e considerando que atualmente o período pré-escolar se restringe aos quatro e cinco anos etários.

\section{Referências}

Almeida, P. M., \& Wickerhauser, H. (1991, abr./ jun.). O critério ABA/ABIPEME - Em busca de uma atualização. Um estudo e uma proposta submetidos à ABA e à ABIPEME. Manuscrito de circulação restrita da Associação Brasileira de Anunciantes e Associação Brasileira dos Institutos de Pesquisa de Mercado, São Paulo, SP, Brasil.

Angelini, A. L., Alves, I. C. B., Custódio, E. M., Duarte, W. F., \& Duarte, J. L. M. (1999). Manual: Matrizes Progressivas Coloridas de Raven. São Paulo, SP: Centro Editor de Testes e Pesquisas em Psicologia.

Brannon, E. M. (2005, March 1). The independence of language and mathematical reasoning. Proceedings of the National Academy of Sciences, 102(9), 3177-3178. doi:10.1073/ pnas.0500328102

Cantlon, J. F., Platt, M. L., \& Brannon, E. M. (2008). Beyond the number domain. Trends in Cognitive Sciences, 13(2), 83-91. doi:10.1016/j. tics.2008.11.007,

Casas-Fernández, C. (1997). Experience with Health Quality of Life Questionnaire for the Epileptic Child (CAVE). Revista de Neurologia, 25(139), 415-421.
Dehaene, S. (1997). The number sense: How the mind creates mathematics. New York: Oxford University Press.

Dehaene, S. (2001). Précis of the number sense. Mind \& Language, 16, 16-36. doi:10.1111/14680017.00154

Dehaene, S., \& Cohen, L. (1995). Un modèle arithmétique e fonctionnel de l'arithmétique mental. In M. Pesenti \& X. Seron (Eds.), Neuropsychologie des troubles du cálculo e du traitement des números (pp. 191-232). Marseille, France: Solal.

Dellatolas, G., von Aster, M., Braga, L. W., Meier, M., \& Deloche, G. (2000). Number processing and mental calculation in school children aged 7 to 10 years: A transcultural comparison. European Child and Adolescent Psychiatry, 9(2)102110. doi:10.1007/s007870070003

Figueiredo, V. L. M. (2002). WISC III. In J. A. Cunha (Ed.), Psicodiagnóstico $V$ (pp. 603-614). Porto Alegre, RS: Artmed.

Geary, D. C. (1995). Reflections of evolution and culture in children's cognition: Implications for mathematical development and instruction. American Psychologist, 50(1), 24-37. doi:10.1037/0003-066X.50.1.24

Geary, D. C. (2000). From infancy to adulthood: The development of numerical abilities. European Child and Adolescent Psychiatry, 9(Suppl. 2), S11-S16. doi:10.1007/s007870070004

Geary, D. C., Bailey, D. H., Littlefield, A., Wood, P., Hoard, M. K., \& Nugent, L. (2009). First-grade predictors of mathematical learning disability: A latent class trajectory analysis. Cognitive Development, 24(4), 411-429. doi:10.1016/j.cogdev.2009.10.001

Gelman, R., \& Butterworth, B. (2005). Number and language: How are they related? Trends in Cognitive Science, 9, 6-10. doi:10.1016/j. tics.2004.11.004

Gilmore, C. K., McCarthy, S. E., \& Spelke, E. S. (2010, June). Non-symbolic arithmetic abilities and mathematics achievement in the first year of formal schooling. Cognition, 115(3), 394-406. doi:10.1016/j.cognition.2010.02.002

$\operatorname{Kercood}_{2}$ S., Zentall, S. S., Vinh, M., \& Tom-Wright, K. (2012). Attentional cuing in math word problems for girls at-risk for ADHD and their peers in general education settings. Contemporary Educational Psychology, 37, 106-112. doi:10.1016/j.cedpsych.2012.02.001 
Koumoula, A., Tsironi, V., Stamouli, V., Bardani, I., Siapati, S., Graham, A., ...von Aster, M. (2004). An epidemiological study of number processing and mental calculation in Greek schoolchildren. Journal of Learning Disabilities, 37(5), 377388. doi:10.1177/00222194040370050201

Krajewski, K., \& Schneider, W. (2009). Exploring the impact of phonological awareness, visual-spatial working memory, and preschool quantity-number competencies on mathematics achievement in elementary school: Findings from a 3-year longitudinal study. Journal of Experimental Child Psychology, 103, 516-531. doi:10.1016/j.jecp.2009.03.009

Kucian, K., Grond, U., Rotzer, S., Henzi, B., Schönmann, C., Plangger, F., ...von Aster, M. (2011). Mental number line training in children with developmental dyscalculia. Neuroimage, 57, $782-$ 795. doi:10.1016/j.neuroimage.2011.01.070

Libertus, M., Feigenson, L., \& Halberda, J. (2011). Preschool acuity of the approximate number system correlates with school math ability. Developmental Science, 14, 1292-1300. doi:10.1111/ j.1467-7687.2011.01080.x

McCloskey, M., Caramazza, A., \& Basili, A. (1985). Cognitive mechanisms in number processing and calculation: Evidence from dyscalculia. Brain and Cognitive, 4, 171-196. doi:10.1016/02782626(85)90069-7

Moyer, R. S., \& Landauer, T. K. (1967). Time required for judgements of numerical inequality. Nature, 215(109), 1519-1520. doi:10.1038/2151519a0

Mussolin, C., Nys, J., Content, A., \& Leybaert, J. (2014). Symbolic number abilities predict later approximate number system acuity in preschool children. PLoS ONE, 9(3), e91839. doi:10.1371/ journal.pone.0091839

Resnick, L. B. (1989). Developing mathematical knowledge. American Psychologist, 44(2), 162169. doi:10.1037/0003-066X.44.2.162

Santos, F. H. (2002). Memória operacional de crianças normais e com lesões congênitas: Desenvolvimento cognitivo e reorganização cerebral (Tese de doutorado não-publicada, Escola Paulista de Medicina, Universidade Federal de São Paulo, SP, Brasil).

Santos, F. H., Silva, P. A., Ribeiro, F. S., Dias, A. L. R. P., Frigério, M. C., Dellatolas, G., \& von Aster, M. (2012). Number representation in Brazilian children number processing and calcula- tion in Brazilian children aged 7-12 years. The Spanish Journal of Psychology, 15(2), 513-525. doi:10.5209/rev_SJOP.2012.v15.n2.38862

Sekuler, R., \& Mierkiewicz, D. (1977). Children's judgments of numerical inequality. Child Development, 48, 630-633.

Silva, P. A., \& Santos F. H. (2011). Discalculia do Desenvolvimento: Avaliação da Representação Numérica pela ZAREKI-R. Psicologia: Teoria e Pesquisa, 27(2), 169-177. doi:10.1590/S010237722011000200003

Sood, S., \& Jitendra, A. K. (2011). An exploratory study of a Number Sense Program to Develop Kindergarten Students' Number Proficiency. Journal of Learning Disabilities, 46(4), 328346. doi:10.1177/0022219411422380

StatSoft. (2004). STATISTICA [Data analysis software system], version 7. Retrieved from www.statsoft.com

Von Aster, M. (2000). Developmental cognitive neuropsychology of number processing and calculation: Varieties of developmental dyscalculia. European Child and Adolescent Psychiatry, 9(2), 41-58. doi:10.1007/s007870070008

Von Aster, M., \& Dellatolas, G. (2006). ZAREKI$R$ - Batterie pour l'évaluation $d u$ traitement des nombres et du calcul chez l'enfant. Paris, France: Les Editions du Centre de Psychologie Appliquée.

Von Aster, M. G., \& Shalev, R. S. (2007). Number development and developmental dyscalculia. Developmental Medicine \& Child Neurology, 49, 868-873. doi:10.1111/j.1469-8749.2007.00868.x

Wechsler, D. (2002). WISC-III: Escala de Inteligência Wechsler para Crianças: Manual (3. ed.). São Paulo, SP: Casa do Psicólogo.

Weinhold-Zulauf, M., Schweiter M., \& von Aster, M. G. (2003). Das Kindergartenalter: Sensitive Periode für die Entwicklung numerischer Fertigkeiten. Kindheit und Entwicklung, 12(4), 222-230.

Wynn, K. (1992). Addition and subtraction by human infants. Nature, 358, 749-750.

Xu, F., Spelke, E., \& Goddard, S. (2005). Number sense in human infants. Developmental Science, 8, 88-101. doi:10.1111/j.14677687.2005.00395.x

Recebido: 14/04/2012

$1^{a}$ revisão: 08/06/2014

Aceite final: 15/07/2014 